\title{
Flood Detection and Water Monitoring System Using IOT

\author{
${ }^{1}$ Minakshi Roy ${ }^{*}{ }^{2}$ Prakar Pradhan, ${ }^{3}$ Jesson George, ${ }^{4}$ Nikhil Pradhan \\ ${ }^{1}$ Assistant Professor of Dept of Computer Science and Engineering, SMIT, Sikkim, India \\ ${ }^{2,3,4}$ B-Tech student, Dept of Computer Science and Engineering, SMIT, Sikkim, India
}

\begin{abstract}
:
Since we are now currently present in an era of Computing Technology, it is essential for everyone and everything to be connected to the internet. IOT is a technology that brings us more and more close to this goal. Our project comprises of smart water monitoring system which is a small prototype for flood detection and avoidance system. This paper explains the working and the workflow of all the components present inside our project. The sensors sense the environment and sends real-time data to the cloud (firebase cloud) and users can view and access this data via their mobile platform. The model gives a warning after the water level rises to a particular height. Since it is a small scaled prototype for flood detection and avoidance system, the working of this model is good. The data are uploaded and changed in the cloud in precision to the sensor and real-time changes in the mobile application is achieved. This model can be used to greatly reduce the casualties in a devastating event of flood.
\end{abstract}

\section{Introduction:}

We are witnessing various drastic advancements in the fields of science and technology over the past few decades. The current industrial age has revolutionized our lives and provides us with plenty of comforts and conveniences. However, this industrial progress has come at a hefty cost of global warming and other environmental disasters such as flood, earthquake, etc. Furthermore, the loss caused by such disasters to life and property is immense.

The increasing carbon footprints and greenhouse gases have severely led to an imbalance and disturbances in the natural cycle of rains and floods. Hence, we are facing the dangers of unwarned and inevitable floods more than ever before.

In order to detect and avoid floods in a timely manner, technology plays a very important role. With the help of the current technology privileges, we can detect and prepare ourselves for an upcoming disaster.

Studies show that such an initiative can really come in handy. In a very recent US flooding due to storms in the Midwest, loss of life and property damage were minimized due to the emergency systems available there. On the other hand, North Korea struggled to deal with the displacement of over 300,000 people, approximately 221 deaths and a cost of $\$ 6$ million- most to feed the homeless survivors, and this all resulted in part from the lack of development of warning systems and information at the community level of the impending flooding. The same was seen in the floods that happened in the Indian states of Kerala and Tamil Nadu.

From the above examples, we may come to the conclusion that an effective warning system is actually a serious problem to developing and underdeveloped countries. A nation like the US has a rich economy and thus, no limitations to resource usage. Warning communities of the incoming flood, therefore, is an expensive proposal given the limited resources of the countries.

The current methods demand for expensive equipment's and centralized, computationally difficult flood detection schemes. This therefore provides an opportunity to use the latest work in information communication technology and sensor networks to solve this problem in such a manner that the overall system balances the minimal cost 
requirement and limited computational power with the need for high reliability of both the system and the computation.

This is where the Internet of Things (IoT) proves to be a very efficient approach. Internet of Things or IoT basically refers to the wireless network between objects embedded with some electronics, software and sensors with the internet, enabling them to collect and exchange data, and can be remotely monitored and controlled.

An IoT early flood detection and alert system using the Arduino is thus, a proposed solution to this problem. The system consists of various sensors which are temperature, humidity, water level, flow and ultrasonic sensors and also includes an Arduino controller, a Wi-Fi module, an LCD, an IoT remote server-based platform and an android application with constructed user friendly GUI relaying all the vital information involved in the picture in a visual format.

The above-mentioned sensors measure the various environmental and weather-related parameters and monitor them constantly. The data from these sensors is constantly fed to an Arduino controller. The Arduino program checks for ant irregularities in the sensor measurements and performs the associated computations. The Arduino also has a Wi-Fi module attached to it, which enables it to send the sensor data to the remote IoT platform using the IoT protocols over the Wi-Fi connection. The LCD is used to display the real-time values of the sensors. These data can also be viewed on the android application, which constantly retrieves the information from the remote IoT platform. If the value of any sensor crosses over a certain threshold value, an alert is sent to the end user via the android application. Using this system, the flood-related parameters can be monitored from anywhere in the world remotely.

Flooding is usually brought on by an increased quantity of water in a water system, like a lake, river overflowing. On occasion a dam fractures, abruptly releasing a massive quantity of water. The outcome is that a number of the water travels into soil, and 'flooding' the region. Rivers are involving riverbanks, in a station. Aside from lack of products and house and office property, streets infrastructure flood water consists of bacteria and sewage flow of waste sites and chemical spillage which leads to a variety of diseases afterwards.

Flood predictions need information like:

i) The speeds of change in river stage on a Realtime basis, which may help indicate the seriousness and immediacy of this threat.

ii) Understanding of the form of storm generating the moisture, such as length, intensity and areal extent, which is valuable for discovering potential seriousness of the flood.

In this system we make use of an Arduino with sensors to predict flood and alert respective authorities and sound instant alarm in nearby villages to instantly transmit information about possible floods using IOT. Different sensors namely flow, ultrasonic, float, temperature, humidity sensors are used to monitor the water level our system utilizes the power of real-time database (firebase) integrated with android.

\section{Implementation method}

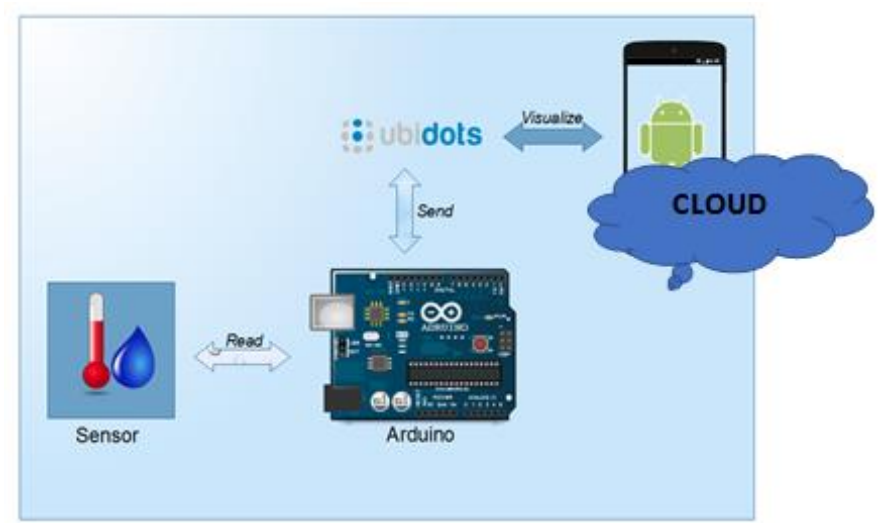

Figure1:

The main idea in our project is to integrate two platforms android and iot in order to realize a system that is dependable of easy to access at the same time.

We use Arduino and different sensors in order to collect and upload the data to a real time database and android to use this data for monitoring purpose.

\section{Hardware implementation}

Different hardware used in our projects are:

i) Arduino Wemos D1 R1.

ii) Ultrasonic sensor.

iii) Flow sensor.

iv) Dht11(Temperature and humidity sensor). 
v) Float sensor.

vi) LCD Display.

i) The Arduino is the heart of the system all the sensors are connected to the Arduino and they operate in a synchronized manner. Arduino Wemos D1 R1 has a built in wi-fi module which is used to upload the data to the database (data is uploaded every 1 second).

ii) Ultrasonic sensor is used to measure the amount of water or the distance from the sensor to the water level.

iii) Flow sensor is used to measure the flow and the amount of water transferred.

iv) DHT11 sensor for measuring temperature and humidity.

v) LCD for displaying the data.

vi) Float sensor acts as a switch when the water level hits the float sensor it causes the float sensor to move up connecting the circuit and hence sends a stress signaling alarming the user for possible rise in water level.

\section{Software implementation}

For software part we have integrated our system with android we developed an android system that is fully capable of monitoring the system environment.

This is done using the data sent by the system. Using an android app makes it for mobile and convenient to use as the app is just one click away. Every detail related to the system can be viewed in this app. It can notify the user if there is any possibility of flood to occur.

For e.g. certain rise in water flow increasing the overall water level of the dam/container (in our case) the application is versatile enough to let the user know about this and report it before hand in order to avoid any form of casualties.

The application can also to be used to monitor sensor data in real time that is it can provide:

i) Distance from ultrasonic sensor to water.

ii) The flow rate of water.

iii) Total volume of water and iv) Temperature and humidity.

All these features provided by the application can be efficiently used by any individual to monitor the system. It is user friendly and avoids complication of different data used as the user is only provided with what really is important.

\section{Conclusion}

Disasters, as the name suggests, brings about great havoc on lives and property indiscriminately across the globe. Developing countries, however, experience much worse destruction than the developed ones and are as well as less equipped to deal with the aftereffects of these calamities. Foreknowledge of the disaster could thus help all, especially the developing countries by providing time to secure property and evacuate. Developing early warning systems may be complicated, with many facets to the system requirements and many additional intricacies, when within a developing country. This paper has tried to propose a potential and economic solution to the problem of floods. Floods cannot be predicted easily, but we are trying to develop a system which tries to detect flood and give early intimation to nearby people. The IoT based flood detection and alert system may prove to save the lives of people by reducing the human quick out during emergency situations. Development of a wireless sensor network has been successfully carried out, with considerations on area of deployment and efficiency. So far, we have built a micro-model through a prototype; the sensors utilized were fundamental in obtaining the required data necessary for monitoring and detecting flood events, and a live feed has also been actualized for end users. The proposed system can later be used to provide solutions to real-life challenges, thereby bringing relief to people in communities ravaged by persistent flood occurrences.

\section{Reference}

Elizabeth Goodman, Martin Charlier, Ann Light \& Alfred Lui "Designing the Internet of Things by Claire Rowland", google books, published in 2015. 